Erschienen in: Ökonomie als Grundlage politischer Entscheidungen : essays on growth, labor markets, and European integration in honor of Michael Bolle / Gabriel, Jürgen et al. (Hrsg.). - Opladen : Leske \& Budrich, 2001. - S. 61-84. - ISBN 3-8100-3317-0

\section{Soziales Kapital und wirtschaftliches Wachstum: ,Vertrauen' als Determinante der Leistungsfähigkeit der EU-Regionen}

Thomas Plümper und Gerald Schneider

Universität Konstanz

\section{Einleitung}

Forschungsfragen ${ }^{1}$ und Forschungsergebnisse sind Moden unterworfen. Als sich Michael Bolle als Assistent an der Freien Universität Berlin Ende der sechziger Jahre mit der Problematik des wirtschaftlichen Wachstums befasste, war nahezu jeder Ökonom Keynesianer. Angesichts der auch in den Wirtschaftswissenschaften vorherrschenden Steuerungseuphorie analysierte Michael Bolle ,optimales Wachstum bei endlichen Zeithorizonten' (Bolle 1969a) und ,optimale Staatsausgabenpolitik unter Verwendung quadratischer und zeitminimaler Gütekriterien' (Bolle 1969b). Ausdruck der damaligen Zeit war das Ergebnis Bolles, dass konjunkturelle Schwankungen durch den Einsatz optimaler Politik, also durch Konjunktursteuerung mittels Steigerung und Verringerung des Staatshaushaltes, „eliminiert werden “ (Bolle 1969b: 354). Dieser Optimismus ist heute bekanntlich verflogen, auch wenn die neuere Wachstumstheorie nach Jahren der Kritik am politischen Interventionismus den möglichen Einfluss von gezielten Infrastruktur-, Humankapital- und F\&E- Ausgaben zur langfristigen Stimulierung des Wirtschaftswachstums betont (Aghion/ Howitt 1998). Eine neuere Entwicklung in diesem Forschungszweig stellt auch die Hinwendung zu kulturalistischen Erklärungen dar. In diesem Zusammenhang fand in den letzten Jahren besonders die These des amerikanischen Politikwissenschaftlers Robert Puinam (1993, 2000) große Beachtung, wonach ,soziales Kapital' in der Form von zwischenmenschlichem Vertrauen eine zentrale Wachstumsdeterminante sei. Anhand einer Analyse der italienischen Regionen bestätigte der amerika-

${ }^{1}$ Dieser Aufsatz stellt eine Erweiterung der Analyse dar, die wir in einer mit Steffen Baumann veröffentlichten Studie erstmals vornahmen (Schneider, Plümper und Baumann 2000). Wir möchten Christina Schneider für ihre Hilfe bei der Erstellung des Manuskripts danken. Michael Neugart, Paul Whiteley und Margret Levi danken wir für ihre Kommentare. 
nische Politikwissenschaftler seine These, wonach diese gesellschaftliche Ressource die wirtschaftliche Entwicklung entscheidend fördere. Noch ist ein Urteil zu früh, ob die Aufwertung, weicher Einflussfaktoren in der Wachstumsdiskussion sehr viel mehr ist als einer jener kurzlebigen Trends, den die akademische Zunft und das politische Feuilleton immer wieder erfasst. Angesichts der Popularität der These von den vorgeblich segensreichen Wirkungen des sozialen Kapitals scheint es uns aber angeraten, die ,Vertrauenshypothese ${ }^{t}$ einer empirischen Evaluation zu unterziehen, die sich möglichst eng an das Forschungsdesign Putnams hält. Wir werden deshalb in diesem Aufsatz die regionale Betrachtungsweise Putnams beibehalten, die Forschungsperspektive aber über Italien hinaus auf den EU- Raum erweitern und in einem multivariaten Design untersuchen, ob das interpersonale Vertrauen und andere Facetten der ,politischen Kultur' wachstumsfördernd sind. Vor dieser empirischen Überprüfung werden wir die theoretischen Grundlagen zu dieser kulturalistischen Erklärung durchleuchten und basierend auf Knack und Zak (2001) ein formales Modell präsentieren, das den Einfluss von Vertrauen auf das wirtschaftliche Wachstum begründet. Anschließend transferieren wir dieses Modell in ein Mankiw-Romer-Weil-Wachstumsmodell und leiten einen Schätzansatz ab, den wir empirisch überprüfen. Unsere Ergebnisse zeigen, dass Vertrauen - wie von Knack und Zak prognostiziert - zwar einen positiven Einfluss auf die Investitionsquote besitzt, dass jedoch die durchschnittliche Faktoreffizienz der Region sinkt. Unklar bleibt, ob der Rückgang der Produktivität direkt auf interpersonales Vertauen zurückzuführen ist oder eher eine Konsequenz der Vergrößerung des Kapitalstocks in notwendigerweise relativ unproduktiveren Unternehmen und Sektoren ist.

\section{Kultur, Sozialkapital und Wachstum in der wissenschaftlichen Diskussion}

Seit den klassischen Abhandlungen Webers ([1921] 1972) über die Rolle des Calvinismus für wirtschaftliches Wachstum spielte das Konzept der ,Politischen Kultur ${ }^{6}$ in den Sozialwissenschaften eine große Rolle. Heute finden sich nur wenige Studien über den Einfluss der Religion auf das Wachstum (Lal 1998), dagegen wurden in den letzten Jahren und Jahrzehnten eine größere Zahl von Untersuchungen zur Bedeutung des Sozialkapitals für den Entwicklungsprozess veröffentlicht.

Klassisch ist in diesem Zusammenhang etwa Kenneth Arrows Aufsatz ,Gifts and Exchanges' (Arrow 1972). Den gegenwärtigen Boom ausgelöst hat jedoch die Monographie ,Making Democracy Work ${ }^{4}$, die Putnam in Zusammenarbeit mit zwei Kollegen 1993 publizierte. Eine der Hauptthesen des vielgelobten Werkes ist, dass interpersonales Vertrauen in der Form von, sozialem Kapital' für die langfristige Entwicklung von Volkswirtschaften von zentraler Bedeutung sei. Diese gesellschaftliche Ressource kann nach Auffassung Putnams helfen, jene Probleme des kollektiven Handelns zu überwinden, die einer wirtschaftlichen Entwicklung abträglich sind. Die Logik des Arguments geht dahin, dass Transaktionskosten bei der Produktion öffentlicher Güter sinken, wenn Vertrauen ohne funktionale Einbuße soziale Kontrolle und Sanktionsandrohungen ersetzt. Darüber hinaus identifiziert Putnam soziale Netzwerke im Anschluss an die bahnbrechenden Arbeiten Colemans (1991) als Bedingung der wirtschaftlichen Entwicklung. Da es in Hierarchien an Kontrolle der jeweils Vorgesetzten mangelt, sind nach Putnam horizontal strukturierte Netzwerke der Ausbildung von sozialem Kapital zuträglicher. Bei den Interaktionen zwischen Gleichgestellten sind nach Putnam in Anlehnung an James Coleman (1998: 103-104) drei Dimensionen von Vertrauen wesentlich: erstens Vertrauen in das reziproke Verhalten eines Interaktionspartners, zweitens Vertrauen in Informationen, die andere Personen zur Verfügung stellen, und drittens Vertrauen in die Funktionsweise sozialer Normen.

Nach Putnam (1993) sind solche horizontal strukturierten Netzwerke in Venetien oder der Toskana zu finden, während das Mezzogiorno unter dem Einfluss hierarchischer Orgainsationen wie der Mafia oder der katholischen Kirche leide. ,Making Democracy Work ${ }^{k}$ zeigt aufgrund dieser Bestandsaufnahme, dass Regionen mit flachen gesellschaftlichen Hierarchien das Vetrauen der Bürger zueinander stärker ausgeprägt sei, dass die politischen Institutionen besser funktionierten und dass letztlich auch das Wirtschaftswachstum überdurchschnittlich ausfalle. Verschiedenste Autoren haben diese eingängigen kommunitaristischen Thesen aufgenommen. Während das politische Feuilleton die Studie Putnams fast uneingeschränkt lobte, war aber auch Kritik zu vernehmen (Schneider, Plümper und Baumann 2000 bieten ausführlichere Literaturhinweise).

Wie tragfähig die Thesen Putnams sind, ist somit noch weitgehend unklar. Keine Einigkeit herrscht etwa zur Frage, ob die Ausstattung eines Gebietes mit politischem Kapital eher über Umfragedaten oder über Verhaltensindikatoren zu messen sei. Ferner ist umstritten, inwieweit das soziale Kapital nicht mehr als nur ein gesellschaftliches Epiphänomen darstellt. Neuste Experimente stützen hier etwa die Erkenntnis, dass soziale Nähe das interpersonale Vertrauen stärkt. Allerdings weisen Gla- 
ser et al. (2000) auch darauf in, dass Personen mit höherem sozialen Status mehr soziales Kapital wecken können als Experimentteilnehmer mit geringerem Prestige. Helliwell und Putnam (1999) zeigen in einer Reanalyse von amerikanischen Umfragedaten, dass das Bildungsniveau eine wesentliche Determinante für die Ausbildung von sozialem Kapital ist.

Putnam geht in seiner neueren monumentalen Monographie (Putnam 2000) ausschnittweise auf solche konzeptuellen Fragen ein, beschränkt sich aber auf eine Analyse der Vereinigten Staaten. Er beklagt für die führende Wirtschaftsmacht aufgrund einer Vielzahl eher deskriptiver Befunde, dass sich das zivile Engagement der Bürger in einem besorgniserregenden Ausmass zurüickgegangen sei. Diese Individualisierungs- und Atomisierungstendenzen kritisiert Putnam wortreich mit dem titelgebenden Schlagwort ,Bowling Alone'. Im Sinne seines ersten Werkes zum Thema wäre an sich zu vermuten, dass die Wohlfahrt unter der dadurch herbeigeführte gesellschaftliche Krise leidet. Leider bleibt in ,Bowling Alone' unerörtert, warum die USA trotz des Niedergangs des Sozialkapitals einen eindrücklichen wirtschaftlichen Aufstieg erlebten.

Dass dieser Kontrast zwischen wirtschaftlichem Erfolg und Verlust an interpersonalem Vertrauen möglicherweise mehr ist als eine, wenn auch bedeutende Ausnahme, verdeutlicht eine Untersuchung Jan van Deths (2000). Er zeigt anhand westeuropäischer Daten, dass soziales Kapital keineswegs das zivile und politische Engagement der befragen Individuen fördert, wie Putnam voraussetzt. Angesichts auch anderer eher konträr zu Putnam (1993) stehender Re-Ananalyse (Jackman/ Miller 1996a, 1996b) erfährt das Konzept des ,sozialen Kapitals' in der wissenschaftlichen Diskussion derzeit eine Neudefinition. So versuchen Guiso, Sapienza und Zingales (2000) den Einfluss des sozialen Kapitals auf verschiedene ökonomische Aktivitäten wie zum Beispiel den informellen Kreditmarkt oder den Gebrauch von Schecks für finanzielle Transaktionen durch die Teilnahmerate bei den italienischen Provinzwahlen zu messen. Vor allem als Test der Hypothesen Putnams scheint die Verwendung von solchen Verhaltensindikatoren insofern ungeeignet, als dass Putnams ursprüngliche Studie Unterschiede im gesellschaftlichen Engagement gerade auf die Differenzen in der Sozialkapitalausstattung zurückführte ${ }^{2}$.

${ }^{2}$ Aus diesem Grund wählen wir in dieser Studie den alternativen Weg der Operationalisierung und bestimmen die Ausstattung von sozialem Kapital über aggregierte Umfragedaten. Wir beziehen uns dabei ausschließlich auf die zweite Hauptthese Putnams, nämlich die angeblich positiven Wirkungen des interpersonalen Vertrauens auf das Wirtschaftswachstum.
Während Putnam seine Hypothesen zur Entwicklung italienischer Regionen mit anekdotischer Evidenz und bivariaten Korrelationsanalysen zu belegen suchte, analysieren einige der Nachfolgestudien den Zusammenhang zwischen Sozialkapital und Wachstum im Rahmen eines einfachen Wachstumsmodells und mit Hilfe multivariater Regressionsmodelle. In den meisten Studien dient dabei der Nationalstaat als grundlegende Untersuchungseinheit. Paul Whiteley $(1998,2000)$ untersucht zum Beispiel 34 Länder in einem Zeitraum von 22 Jahren und zeigt, dass ein neoklassisches Wachstumsmodell entscheidend an Aussagekraft gewinnt, wenn es kulturelle Variablen in die Analyse einbezieht. Eine hohe Sozialkapitalausstattung fördert folglich wirtschaftliches Wachstum ungeachtet der ökonomischen Struktur oder dem Grad der Demokratisierung, der das politische System charakterisiert.

Die Einblicke, die aus den Arbeiten von Granato et al. (1996a; 1996b), Jackman und Miller (1996a; 1996b) und Swank (1996) gewonnen werden können, sind hingegen weniger eindeutig als die Resultate Whiteleys. Während Granato et al. (1996a; 1996b) im Anschluss an Ingleharts Studien zum Postmaterialsmus die Thesen Putnams auf ein breites Sample von Staaten zu übertragen versuchen und so mit Standardkonzepten der Politischen Soziologie verbinden, weist Swank (1996) auf die Bedeutung einiger weiterer ,kultureller' Faktoren wie Korporatismus und Konfuzianismus hin. Jackman und Miller (1996a: 635) dagegen glauben, dass Kultur keine systematischen Auswirkungen auf wirtschaftliches Wachstum ausübt. Das Autorenpaar schließt daraus, dass jede weitere Anstrengung, das Konstrukt politische Kultur' zu definieren und zu testen wenig produktiv sei (1996a: 654) ${ }^{3}$. Ihre Hauptkritik an den anderen Studien bezieht sich zum größten Teil auf die grobe Messung von Kultur durch Dummyvariablen.

Dass die Diskussion über die Wirkung kultureller Faktoren auf wirtschaftliches Wachstum noch zu keinem klaren Ergebnis geführt hat, könnte zum einen tatsächlich in den unterschiedlichen operationalen Definitionen der zentralen Konzepte wie ,politische Kultur" oder ,soziales Kapital" liegen. Putnam und Inglehart zum Beispiel sehen die Ausstattung einer Gesellschaft mit sozialem Kapital als ein schon weitgehend gegebenes strukturelles Attribut an, während Rationalisten wie etwa Coleman (1988) oder Jackman und Miller (1996a, b) das Konzept als Konsequenz anderer Merkmale einer Gesellschaft begreifen. Zum anderen

${ }^{3}$ Eine ebenso negative Bewertung erfahren in diesem Zusammenhang die $\mathrm{Hy}-$ pothesen Putnams, dass über soziales Kapital die Qualität von politischen Institutionen wachse. 
beruhen die empirischen Analysen auf unterschiedlichen Untersuchungseinheiten. Während Putnams ursprüngliche Analyse auf einem Vergleich der italienischen Regionen beruht, liegt der analytische Fokus der Nachfolgestudien zumeist auf dem entwickelten Industriestaat - und damit auf einem Aggregat, das bezüglich seiner kulturellen Identität wohl weniger homogen ist, als man bei einer Regressionsanalyse eigentlich voraussetzen müsste.

Unsere Studie versucht die Diskussion insofern voran zu treiben, als wir uns in der multivariaten Überprüfung auf die gleiche Untersuchungseinheit wie Putnam stützen. Zugleich verwenden wir in Übereinstimmung mit Whiteley (1998, 2000), aber in Abgrenzung zu den meisten anderen politikwissenschaftlichen Beiträgen ein dezidiertes ökonomisches Wachstumsmodell als Ausgangspunkt.

In Übereinstimmung mit der neueren Wachstumsliteratur kontrollieren wir für das Wachstum des regionalen Kapitalstocks und das wirtschaftliche Aufholen ärmerer Regionen (Barro und Sala-i-Martin, 1992). Der Begriff der Konvergenz lässt sich auf Gerschenkron zurückführen, der als erster ökonomische Rückständigkeit systematisch untersucht hat. In dieser Konvergenzperspektive wachsen ärmere Länder schneller als reichere. Darüber hinaus kontrollieren wir in unserer Studie nationale ökonomische Bedingungen wie zum Beispiel den Grad der wirtschaftlichen Öffnung und den Regierungsanteil am BIP.

Nach Auffassung neuerer Forschungsliteratur stellen die europäischen Regionen einen klassischen Konvergenzklub dar. Barro und Sala-i-Martin (1995) haben zum Beispiel gezeigt, dass die unkonditionale Beta-Konvergenz innerhalb der europäischen Regionen bei etwa 2 Prozent liegt und langfristig stabil ist. Dies bedeutet, dass sich in 35 Jahren die ursprünglichen Einkommensunterschiede um 50 Prozent und in 115 Jahren um 95 Prozent reduziert haben sollten ${ }^{4}$.

Auf jeden Fall hat die neue Wachstumstheorie gezeigt, dass Wachstum nicht nur allein durch ökonomische Faktoren bedingt ist (Aghion und Howitt, 1998). Vielmehr sollte die Faktoreffizienz - also Humankapital und Produktivität - berücksichtigt und ausgehend von politischen und sozio-ökonomischen Faktoren endogenisiert werden. Hansson und Henrekson (1997) sind etwa der Ansicht, dass eine ausreichende Ausstattung mit sozialem Kapital eine Grundvoraussetzung für die von der Neoklassik seit Solow (1956) und Swann (1956) postulierten wirtschaft-

\footnotetext{
${ }^{4}$ Neven und Gouyette (1995) kommen in ihrer Analyse zum Schluss, das Binnenmarktprogramm habe den Aufholprozess verlangsamt. Armstrong (1995) bestätigt, dass der Prozess in den 60 er Jahren stärker ausgeprägt war.
}

lichen Aufholprozesse ist. Aus einer theoretischen Perspektive bleibt es bis jetzt aber unklar, wie kulturelle Faktoren die Faktoreffizienz beeinflussen. Der folgende Abschnitt skizziert auf der Grundlage der Arbeit von Knack und Zak, wie sich kulturelle Faktoren, wie etwa das soziale Kapital, in die neue Wachstumstheorie eingliedern lassen ${ }^{5}$.

\section{Vertrauen und Wachstum: Eine Variante des Knack-Zak-Modells}

Das Knack-Zak-Modell (2001) geht von der Existenz heterogener Individuen (mit $i$ indiziert) aus. Die Unterschiede zwischen den Individuen lassen sich auf einer Dimension abtragen. Alle Akteure besitzen standardisierte Präferenzen und sparen, um ihren Konsum intertemporal zu glätten. Um jedoch Zugang zum Kreditmarkt zu bekommen, müssen sie einen ,Broker" (mit $j$ indiziert) in Anspruch nehmen, der ihre individuellen Ersparnisse in den Kreditmarkt einspeist. Der Broker kennt im Gegensatz zum Sparer die Rendite der unterschiedlichen Geldanlagen. Aufgrund dieses Informationsvorsprungs ist es dem Broker prinzipiell möglich, den Vermögensbesitzer zu betrügen. Allerdings variiert die Befähigung zum Betrug zwischen den Brokern, und der Anleger kann Informationen über die Betrugswahrscheinlichkeit der unterschiedlichen Broker sammeln. Diese Informationssammlung ist freilich transaktionskostenintensiv. Bekannt ist dagegen der relative Anteil der betrügerischen Broker an allen Anlageberatern.

Knack und Zak ergänzen dieses einfache Prinzipal-Agent-Modell um institutionelle Gesichtspunkte: Die erste von ihnen eingeführte Regel besteht darin, dass eine Organisation wie beispielsweise die Bundesanstalt für das Kreditwesen Informationen über die Betrugswahrscheinlichkeit der einzelnen Broker veröffentlichen kann. Eine zweiten Regel besagt, dass betrügerische Broker sanktioniert werden können, wenn die Anleger Steuern zahlen, die eine gerichtsähnliche Institution finanzieren, die den Brokern deren Beratungsgebühren entziehen kann.

\footnotetext{
${ }^{5}$ Auch einige andere theoretische Beiträge vermögen überzeugend zu argumentieren, wie soziales Kapital das Wirtschaftswachstum beeinflusst. Dies gilt etwa für den Beitrag von Hillman und Ursprung (2000), welche den neuen Zweig in der Wachstumsliteratur mit einer Rentseeking-Perspektive verknüpfen. Bisin und Verdier (2000) wiederum beschäftigen sich mit Sozialisationseffekten, ohne genau die Wirkungen solcher sozialer Prozesse auf wirtschaftliche Entscheidungen zu zeigen.
} 
$\mathrm{Zu}$ den juristisch geregelten Bestrafungsmethoden gesellen sich ferner soziale Sanktionen. So müssen die Betrüger mit einem gesellschaftlichen Reputationsverlust rechnen, da sie mit ihrem Verhalten das interpersonale Vertrauen geschädigt haben. Je besser die gesellschaftliche Kommunikation und je mehr sich die an den Transaktionen beteiligten Individuen vertrauen, desto stärker wirkt sich ein Reputationsverlust auf den Umsatz und die Gewinne der Broker aus. Alle drei Mechanismen reduzieren den Anreiz der Broker, ihren Informationsvorsprung zur Maximierung ihres eigenen Nutzens einzusetzen.

Im Knack-Zak-Modell ist die Informationsasymmetrie zwischen Prinzipal und Agent, also zwischen den Anlegern $i$ und den Brokern $j$, definiert als ,Distanz' $0 \leq d_{i j} \leq 1$. Diese Distanz wirkt sich auf die Wahrscheinlichkeit des Anlagebetrugs durch den Broker aus. Wenn $d$ klein ist, ist die Betrugswahrscheinlichkeit gering; mit zunehmenden $d_{i j}$ steigt die Betrugswahrscheinlichkeit an.

Nun sei der Nutzen der Anleger $i$ eine Funktion ihres Stundenlohns, der gearbeiteten Zeit, ihres Vermögens, der Besteuerung und ihres Kapitals

$$
U_{i}=f\left(c_{i}, w_{i}, a_{i}, h_{i}, t_{i}\right)
$$

wobei $c_{i}$ den Konsum des Anlegers, $w_{i}$ seinen Stundenlohn, $a_{i}$ sein Vermögen und $h_{i}$ seine Arbeitszeit darstellt. Dabei müssen die Anleger die ihnen zur Verfügung stehende Zeit auf die Arbeit und die Pflege ihres Vermögens aufteilen

$$
1=h_{i}+e_{i}
$$

$e_{i}$ bemisst die Dauer, die der Akteur zur Evaluierung der Anlageoptionen aufbringt. Des weiteren können die Investoren eine Steuer $t_{i}$ aufbringen, um eine Institution $p$ zu finanzieren, welche die Broker überwacht und gegebenenfalls sanktioniert. Die Steuer senkt naturgemäß den Nutzen der Anleger.

Ein funktionales Äquivalent für die Überwachungsagentur $p$ und die individuelle Sammlung von Informationen $e$ stellt das soziale Kapital $\xi$ dar. Je höher $\xi$, desto geringer wirkt sich die Distanz zwischen Investor und Broker, $d_{i j}$, aus. Die wirksame Distanz $D$ ist folglich

$$
D_{i j}=\frac{d_{i j}}{\xi}
$$

wobei $\xi \geq 1$. Wenn das soziale Kapital gegen unendlich strebt, geht die wirksame Distanz gegen Null. Wenn das soziale Kapital minimal ist $(=1)$, dann ist die wirksame Distanz gleich der tatsächlichen Distanz.
Die Kenntnis der Anleger über den Charakter der Broker folgt somit

$$
\eta=f\left(e_{i}, p, D_{i j}\right)
$$

für alle $0 \leq \eta \leq 1$.

Die Anleger verteilen ihre Zeit auf lohnabhängige Arbeit und das Sammeln von Informationen über ihren Broker. Dann wählt der Akteur seine individuelle Sparquote und übergibt dieses nicht-konsumierte Einkommen an einen der Broker.

Das Problem des Akteurs $i$ zum Zeitpunkt $t$ besteht nun darin, seinen Lebenskonsum unter den folgenden Randbedingungen zu maximieren:

$$
c_{i, t}=w_{i, t} h_{i, t}+(1+r-\delta) a_{i, t} \eta_{i, j}-\left(a_{i, t}-a_{i, t-1}\right)-t_{i, t}
$$

wobei $r$ der Zins und $\delta$ die Inflationsrate ist. Das Individuum konsumiert also aus seinem Arbeitseinkommen $w_{i, t} h_{i, t}$ und seinem Kapitaleinkommen $(1+r-\delta) a_{i, t} \eta_{i, j}$ abzüglich der Veränderung seines akkumulierten Vermögens $a_{i, t}-a_{i, t-1}$ und abzüglich der Steuer $t_{i, t}$. Dabei ist die Annahme der eigentlichen Maximierungsfunktion für das Knack-Zak-Model unerheblich. Knack und Zak gehen (wie bereits eingangs erwähnt) davon aus, dass die Individuen heterogen sind, aber dass sie sich lediglich im Hinblick auf ihre Bereitschaft zu sparen unterscheiden. Die Verteilung der Sparpräferenz in der Population ist ebenfalls unerheblich, solange zumindest einige Akteure eine Sparquote größer Null aufweisen.

Um das Modell einfach zu halten, nehmen Knack und Zak (2001) an, dass Broker risikoneutral sind und ihrerseits nicht sparen. Der Konsum des repräsentativen Brokers $j$ hängt vom Kapitalstock des Investors $i$ sowie dem Kenntnisstand des Anlegers über den Broker ab:

$$
c_{j, t}=1-\eta_{i j}(1+r-\delta) a_{i, t}
$$

Wenn der Anleger den Broker perfekt einzuschätzen weiß, ist der Konsum des Brokers folglich Null. Dieser Zustand ist für die Anleger optimal. Für die Ökonomie ist dagegen optimal, wenn $\eta$ nicht nur gleich 1 ist, sondern wenn der Anleger auch keine Transaktionskosten aufbringen muss, um den Zustand $\eta=1 \mathrm{zu}$ erreichen. In diesem Sinne betrachtet Gleichung 3 den Kenntnisstand $\eta$ als Folge des Beobachtungsaufwandes $e_{i}$, der Effektivität der Institution $p$ sowie der wirksamen Distanz, die immer dann gegen Null geht, wenn das soziale Kapital gegen Unendlich strebt. Man beachte nun, dass $e_{i}, p$ und $\xi$ perfekte Substitute sind, dass jedoch nur das soziale Kapital $\xi$ für den Akteur $i$ unendgeldlich zu haben ist. $p$ kostet Steuern $t_{i}$ und $e_{i}$ geht auf Kosten der Arbeitszeit. Ein individuelles 
Wohlfahrtsoptimum ist somit nur dann erreicht, wenn das Sozialkapital gegen unendlich geht, weil dann die wirksame Distanz gegen Null geht.

\section{Ableitung des Schätzansatzes aus einem endogenen Wachstumsmodell}

In diesem Abschnitt integrieren wir dieses theoretische Modell in ein ,endogenes ' Wachstumsmodell des Mankiw-Romer-Weil-Types (1992). Demnach sei

$$
Y_{r, t}=K_{r, t}^{\alpha} H_{r, t}^{\beta}\left(A_{r, t} L_{r, t}\right)^{1-\alpha-\beta}
$$

wobei $Y$ für das Bruttoinlandsprodukt steht, $K$ dem Kapitalstock entspricht, $H$ das Humankapital darstellt, $A$ die Faktoreffizienz misst, und $L$ die Arbeit erfasst. Die Subskripte drücken die Parameterwerte für Region $r$ zur Zeit $t$ aus, $\alpha$ und $\beta$ sind Elastizitäten, wobei $0<\alpha+\beta<1$, so dass abnehmende Skalenerträge angenommen werden.

Mankiw, Romer und Weil (1992) nehmen an, dass die Wachstumsraten von $K$ und $L$ exogen bestimmt werden und $n$ beziehungsweise $g$ betragen:

$$
\begin{aligned}
& L(t)=L(0) e^{n t} \\
& A(t)=A(0) e^{g t}
\end{aligned}
$$

Der Wachstumspfad von physischem Kapitalstock und Humankapital folgt

$$
\begin{aligned}
& \partial k_{t}=s_{k} y_{t}-(n+g+\delta) k_{t} \\
& \partial h_{t}=s_{h} y_{t}-(n+g+\delta) h_{t}
\end{aligned}
$$

wobei $s_{k}$ und $s_{h}$ die Anteile am Output sind, die zum Zeitpunkt $t$ in Sach- und Humankapital investiert werden.

Wir betrachten nun die Veränderungsrate des Kapitalstocks als Funktion der wirksamen Distanz, die ihrerseits durch eine höhere Sozialkapitalausstattung verringert wird,

$$
\frac{\partial K}{\partial L}=\frac{\partial \hat{K} \cdot\left(1-D_{i j}\right)}{\partial L}
$$

$\partial \hat{K}$ misst die maximal erreichbare Veränderungsrate des Kapitalstocks.
Teilen wir nun Gleichung 7 durch $L$ und leiten die Funktion ab, so ergibt sich

$$
\begin{aligned}
\partial\left(\frac{y}{L}\right)_{r, t} & =\partial A_{t}+g t-\frac{\alpha+\beta}{1-\alpha-\beta} \partial(n+g+\delta)+\frac{\alpha}{1-\alpha-\beta}\left(\frac{\partial K}{\partial L}\right)_{r, t} \\
& +\frac{\beta}{1-\alpha-\beta}\left(\frac{\partial H}{\partial L}\right)_{r, t}
\end{aligned}
$$

Setzt man nun Gleichung 12 in Gleichung 13 ein, erhält man

$$
\begin{aligned}
\partial\left(\frac{y}{L}\right)_{r, t} & \left.=\partial A_{t}+g t-\frac{\alpha+\beta}{1-\alpha-\beta} \partial(n+g+\delta)+\frac{\alpha}{1-\alpha-\beta}\left(\frac{\partial K}{\partial L}\right)_{r, t}\right) \\
& +\frac{\beta}{1-\alpha-\beta}\left[\frac{\partial \hat{K} \cdot\left(1-D_{i j}\right)}{\partial L}\right]_{r, t} .
\end{aligned}
$$

Soziales Kapital wirkt sich entsprechend des Knack-Zak-Modells auf die individuelle Sparquote und somit im Aggregat auch auf die Investitionsquote aus. Je eher die Broker in der Lage sind, ihre Eigeninteressen gegenüber den Interessen der Anleger durchzusetzen, desto höher ist die Investitionsquote und die sich daraus ergebende Wachstumsrate.

In Unkenntnis der Distanz' zwischen Anleger und Broker kann diese Gleichung durch das folgende empirische Wachstumsmodell (M1, M2) approximiert werden

$\partial\left(\frac{Y}{L}\right)_{r, t}=\alpha+\beta_{1} \ln \left(\frac{Y}{L}\right)_{r, t-1}+\beta_{2}\left(1-\xi_{r, t-1}\right)+\beta_{3} \frac{\partial K_{r, t}}{\partial L r, t}+\beta_{4} \sum X_{r, t}+\varepsilon$

wobei $X_{i, t}$ für einen Vektor aus Kontrollvariablen steht. Um aber nicht nur eine Einschätzung der Auswirkung des Sozialkapitals auf das Wirtschaftswachstum zu erhalten, sondern auch um Aufschluss über die Auswirkung des Sozialkapitals auf die Investitionsquote zu gewinnen, schätzen wir auch das folgende Investitionsquotenmodell (M3, M4)

$$
\partial\left(\frac{K}{L}\right)_{r, t}=\alpha+\beta_{1} \ln \left(\frac{Y}{L}\right)_{r, t-1}+\lambda\left(1-\xi_{r, t}\right)+\beta_{2} \sum X_{r, t}+\varepsilon
$$

Dabei bezieht sich der Schätzansatz eher auf die Hypothese Robert Putnams, dass eine hohe Ausstattung mit Sozialkapital das Wirtschaftswachstum fördert, während Schätzansatz 14 im wesentlichen dem KnackZak-Modell entspricht. Unsere Analyse bezieht sich auf drei generelle 
Hypothesen, welche die Auswirkungen der theoretisch hergeleiteten Determinanten auf den Kapitalstock und das Wirtschaftswachstum in den EU-Regionen zum Inhalt haben. Wir beziehen uns dabei nicht nur auf die Ausstattung einer Region mit ,sozialem Kapital' ${ }^{\star}$, sondern berücksichtigen in Ubereinstimmung mit der Politischen Soziologie die generelle politische Kultur einer Region.

Zur Überprüfung der Hypothese über die Wirkung der politischen Kultur auf wirtschaftliches Wachstum verwenden wir ein typisches OLSWachstumsmodell. Da politische Kultur ein multidimensionales Konzept ist, werden wir uns zur Messung möglicher Wirkungen auf verschiedene Variablen stützen. Dazu beziehen wir Messungen zum Niveau von interpersonalem Vertrauen und dem Vorhandensein von traditionellen Einstellungen zur Erziehung in den Regionen mit ein.

\section{Operationalisierung und Variablen}

Da wir über keine geographischen Längsschnittdaten für die Entwicklung des Sozialkapitals in den EU-Regionen verfügen, werden wir zur Überprüfung unserer Hypothesen eine Querschnittsregression verwenden. Wir werden die Wirkungen verschiedener Prädiktoren bewerten, die sich auf die durchschnittliche Jahreswachstumsrate in 58 europäischen Regionen in der Zeit von 1980 bis 1996 beziehen. Um die Regionen innerhalb der EU zu identifizieren, werden wir uns auf die von der Europäischen Kommission erstellten NUTS II Kategorisierung (Nomenclature des Units Territoriales Statistiques - II) beziehen ${ }^{6}$. Wir haben uns für diese Kategorisierung entschieden, da hier die Einheiten zumeist regionalen politischen Akteuren entsprechen, die über eine gewisse Autonomie verfügen und auch eine eigenständige Identität aufweisen. Die deutschen Bundesländer, die spanischen Communidades Autónomas, die italienischen Regione oder die belgischen Régions sind zum Beispiel NUTS II-Einheiten. Die Europäische Kommission unterschied in der Untersuchungsperiode zwischen 73 NUTS II-Regionen. Bedauerlicherweise berichtet EUROSTAT aber für diesen Zeitraum nicht über regionales

\footnotetext{
${ }^{6}$ Die Europäische Union hat für administrative und statistische Zwecke für jedes Land eine Unterteilung in territoriale Einheiten vorgenommen. Es existieren drei verschiedene Unterscheidungskategorien (NUTS I, II und III). NUTS I beinhaltet die größten territorialen Einheiten, NUTS III die kleinsten. Die Kategorisierungen orientieren sich nicht zwingend an nationalen administrativen Einheiten.
}

Wachstum und Investitionsraten in den Ländern Österreich, Dänemark, Irland, Portugal und Schweden. Darüber hinaus haben wir die ostdeutschen Bundesländer von der Untersuchung ausgeschlossen, da sie erst in den frühen 90er Jahren Teil des westlichen politischen Systems wurden und aus diesem Grund keine verlässlichen Aussagen über das Wachstum vor 1989 vorhanden sind.

Unsere Untersuchung basiert auf Standarddatensätzen. Wir verwendeten den Eurobarometer 44.0, um Daten für die verschiedenen Aspekte der politischen Kultur zu gewinnen. Der Eurobarometer 44.0 (EB 44) entstand zwischen Oktober und November 1995 (Zentralarchiv, 1996). Wir erhoben Durchschnittswerte für vier Indikatoren von politischer Kultur pro Region.

TRUST basiert auf der Frage (V298-V312):, Jetzt würde ich Sie gerne nach Ihrem Vertrauen in Menschen aus verschiedenen Ländern fragen." Hier arbeiten wir nur mit denjenigen Antworten, die uns Informationen über Vertrauen in die eigenen Landsleute geben. Wieder haben wir die originalen Werte der Variablen so kodiert, dass der Wert 0 einer Tendenz zu wenig bis keinem Vertrauen entspricht und der Wert 1 für eine Tendenz zu Vertrauen steht. Wir erwarten einen positiven Wert, das heißt, je mehr die Menschen ihren Landsleuten vertrauen, desto höher sind die Wachstumsraten ${ }^{7}$.

Zuletzt haben wir zwei Variablen miteinbezogen, die Aufschluss über die Meinung der Bevölkerung über die Rolle der Schule geben sollen (V344-V356). „Vorbereitung der Kinder auf das spätere Leben“, „Vorbereitung der Kinder auf den zukünftigen Beruf" und „Lernen im Team zu arbeiten" kennzeichnen eine Orientierung an wirtschaftlichem Wohlstand, also an materialistischen Werten (ECONVAL). Ein positiver Koeffizient kennzeichnet eine positive Wirkung materialistischer Werte auf wirtschaftliches Wachstum. dKAP misst die Veränderungsrate des Kapitalstocks in der jeweiligen Region. Als Kontrollvariablen nehmen wir den nationalstaatlichen Außenhandelsquotienten OPEN und den nationalstaatlichen Regierungskonsum GOV jeweils in der Untersuchungsperiode auf.

${ }^{7}$ Putnam und seine Mitarbeiter haben weitere Indikatoren erhoben, um politische Kultur in den italienischen Regionen zu beschreiben. Sie fanden zum Beispiel heraus, dass in diesem Kontext der Medienkonsum ein wichtiger Aspekt ist. Obwohl der Eurobarometer einige Variablen über verschiedene Informationsquellen aufweist, war es uns nicht möglich, einen Indikator aufzustellen, der die durchschnittlichen Informationsquellen reliabel zusammengefasst hätte. 


\section{Die Wirkung von sozialem Kapital und politischer Kultur}

Ein Blick in die Daten verdeutlicht, dass die ärmeren Regionen Europas, zu denen besonders Portugal, Irland und Süditalien zählen, die höchsten Wachstumsraten verzeichnen. Dagegen weisen schwedische, dänische und die eher reichen britischen Regionen die geringsten Wachstumsraten auf. Bei den Kultur-Variablen ergeben sich zum Teil unerwartete Unterschiede. Am vertrauenswürdigsten scheinen die Deutschen zu sein; das andere Extrem besetzen die Italiener. Zusätzlich überwiegen in Deutschland, in Irland und in Nordirland materialistische Werte, während Italiener eher postmateriell eingestellt sind.

In Tabelle 1 sind die Schätzergebnisse ausgewiesen, die wir bei der statistischen Analyse der vier Modelle erhielten. Die zwei ersten Modelle entsprechen der Auffassung Putnams, dass die Sozialkapitalausstattung eine exogene Wachstumsdeterminante sei. Modell 3 und 4 hingegen betrachten im Sinne unserer eigenen theoretischen Erweiterung die Sozialkapitalausstattung als Faktor, der die Kapitalstockveränderungen beeinflusst. Die Koeffizienten der Variablen der Standardwachstumsmodelle, welche die Putnam-Hypothese prüfen (M1, M2), weisen in die erwartete Richtung. Die untersuchten Koeffizienten der Konvergenzvariablen lnGDPCt-1 erweisen sich in allen Modellen als hoch signifikant. Dabei bestätigt sich die Relevanz der Konvergenz zwischen den Regionen ${ }^{8}$. Unsere Ergebñisse $(-0,02)$ entsprechen exakt den Ergebnissen Barros und Sala-i-Martins für die Konvergenz der europäischen Regionen (Barro/ Sala-i-Martin 1995: 398). Erstaunlicherweise fällt die Konvergenzrate zwischen den europäischen Regionen geringer aus, als sie typischerweise in Ländersamples geschätzt werden, in die Entwicklungsländer und Industriestaaten aufgenommen wurden.

Physische Kapitalakkumulation korreliert ebenso erwartungsgemäB positiv mit Wachstum ${ }^{9}$. Im Unterschied zu den Ergebnissen von Barro und Sala-i-Martin (1995: 425) liegen unsere Koeffizienten höher (0,09 zu 0,07 ), was sowohl auf die geringere Datenqualität der Eurostat- relativ zu den Penn World Table-Daten als auch auf Unterschiede zurückgeführt werden können, die durch das Ländersample verursacht sind.

\footnotetext{
${ }^{8}$ Für eine Diskussion über Konvergenz im Kontext einer Wachstumstheorie siehe Mankiw et al. (1992) sowie Barro und Sala-i-Martin (1995).

${ }^{9}$ Diese Korrelation ist ein bereits etabliertes Faktum in der neuen empirischen

Wachstumsliteratur. Siehe (neben vielen anderen) Barro (1991), Delong und Summers (1991), Levine und Renelt (1992) sowie Mankiw et al. (1992)
}

Tabelle1. Multivariate Regressionsergebnisse

\begin{tabular}{|c|c|c|c|c|}
\hline & \multicolumn{2}{|c|}{\begin{tabular}{|l|} 
Gleichung 13 \\
Wachstumsregression
\end{tabular}} & \multicolumn{2}{|c|}{$\begin{array}{l}\text { Gleichung } 14 \\
\text { Kapitalstockregression }\end{array}$} \\
\hline & M1 & M2 & M3 & M4 \\
\hline $\begin{array}{l}\text { Abhängige } \\
\text { Variable }\end{array}$ & dGDPC & dGDPC & dKAP & dKAP \\
\hline Intercept & $\begin{array}{l}0,143 \\
(6,800) * * * *\end{array}$ & $\begin{array}{l}0,158 \\
(7,232) * * * *\end{array}$ & $\begin{array}{l}0,316 \\
(2,952) * * *\end{array}$ & $\begin{array}{l}0,379 \\
(3,607) * * *\end{array}$ \\
\hline $\ln G D P C_{t 1}$ & $\begin{array}{l}-0,0155 \\
(-2,625)\end{array} * *$ & $\begin{array}{l}-0,0203 \\
(-3,251)\end{array}$ & $\left(\begin{array}{l}-0,089 \\
(-2,986)\end{array} * * *\right.$ & $\begin{array}{l}-0,111 \\
(-3,724)^{* * * *}\end{array}$ \\
\hline dKAP & $\begin{array}{l}0,0994 \\
(3,977) * * * *\end{array}$ & $\begin{array}{l}0,0827 \\
(3,208) * * *\end{array}$ & & \\
\hline GOV & $\begin{array}{l}0,0006 \\
(1,303)\end{array}$ & $\begin{array}{l}0,0006 \\
(1,334)\end{array}$ & $\begin{array}{l}-0,0080 \\
(-3,777)^{* * * *}\end{array}$ & $\begin{array}{l}-0,0072 \\
(-3,488) * * *\end{array}$ \\
\hline OPEN & $\begin{array}{l}0,0001 \\
(1,226)\end{array}$ & $\begin{array}{l}0,0001 \\
(1,596)\end{array}$ & $\begin{array}{l}-0,0003 \\
(-1,253)\end{array}$ & $\begin{array}{l}-0,0001 \\
(-0,705)\end{array}$ \\
\hline TRUST & $-0,0491$ & $-0,0497$ & 0,232 & 0,203 \\
\hline ECONVAL & $(-3,580)+7+$ & $\begin{array}{l}0,0386 \\
(1,956)\end{array}$ & $(3,414) * * *$ & $\begin{array}{l}(3,077) * * * \\
0,252 \\
(2,521) * *\end{array}$ \\
\hline $\mathrm{N}$ & 58 & 58 & 58 & 58 \\
\hline $\operatorname{adj} \cdot R^{2}$ &, 475 &, 503 &, 316 &, 379 \\
\hline RMS-residual & 0,0024 & 0,0022 & 0,0734 & 0,0654 \\
\hline F-Stat & $11,332 * * * *$ & $10,606^{* * * *}$ & $7,580^{* * * *}$ & $7,949 * * * *$ \\
\hline
\end{tabular}

t-Statistik in Klammern; $* 10 \%$ signifikant, $* * 5 \%$ signifikant, $* * * 1 \%$ signifikant,****0,1\% signifikant.

Bei den einzelnen Kulturvariablen ergeben sich interessante Unterschiede. Während das Ausmaß des interpersonalen Vertrauens TRUST eine negative Wirkung auf wirtschaftliches Wachstum besitzt, fördern materialistische Werte die wirtschaftliche Entwicklung. Die statistische Analyse widerlegt also die naive Auffassung, dass die politische Kultur ein einfaches Attribut ist, das direkt das Wachstum fördert.

Wenn wir das ,soziale Kapital' und andere kulturalistische Variablen hingegen als endogene Faktoren begreifen, die zunächst einmal den Kapitalstock verändern, gelangen wir zu einer präziseren Einschätzung der Wirkungen von Kultur auf wirtschaftliche Handlungen. Die Modelle 3 und 4 testen das Knack-Zak-Modell so direkt, wie es mit den verfügbaren Daten möglich ist. Hier bestätigt sich das Modell: Sowohl TRUST als 
auch ECONVAL weisen einen signifikant positiven Einfluss auf die Höhe der Sachkapitalinvestitionen auf.

Wie sind diese Ergebnisse zu interpretieren? Wenn die Veränderung des Kapitalstocks oder die Investitionsquote in den Schätzansatz aufgenommen werden, messen die Koeffizienten der Variablen, die nicht direkt der Produktionsfunktion (Gleichung 7) angehören, den Einfluss der Variablen auf die Faktoreffizienz. Offenkundig besitzt TRUST einen negativen Einfluss auf die durchschnittliche Produktivität der Nettoanlageinvestitionen. Dieser negative Zusammenhang wird durch den starken Einfluss von Trust auf die Investitionsquote erklärlich. Insgesamt bestätigen unsere Ergebnisse das Knack-Zak-Modell, während die Richtigkeit der Putnam-Hypothese in der von Putnam vertretenen radikalen Version bezweifelt werden muss.

\section{Schlussfolgerung}

Unsere Analysen zeigen, dass interpersonales Vertrauen und damit woh die Hauptkomponente des ,sozialen Kapitals“ der wirtschaftlichen Entwicklung nicht zweifelsfrei förderlich ist. Zwar wird einerseits die Investitionsquote erhöht, doch andererseits sinkt die durchschnittliche Faktoreffizienz. Dieses Resultat korrigiert den Optimismus, den Vertreter der Politischen Soziologie wie Robert Putnam hegen. Ihrer Auffassung nach ist eine entwickelte politische Kultur ein zentraler Wachstumsmotor. Demgegenüber bestätigen unsere Resultate die Befunde der neuen Wachstumsmodelle, die ja immer auch die Bedeutung von politischen Einflussfaktoren betonte. Wir glauben, dass zu diesen Faktoren in $\mathrm{Zu}$ kunft auch kulturelle und institutionelle Faktoren hinzugefügt werden können, wobei allerdings nur Modelle, die tiefer greifen als monokausale Erklärungen, in diesem Zusammenhang adäquat sind. Dieser Aufsatz hat skizziert, in welcher Richtung sich eine an die Ökonomie anschlussfähige kulturalistische Wachstumstheorie entwickeln könnte.

\section{Literatur}

Aghion, P.; Howitt P. (1998): Endogenous Growth Theory. Cambridge: MIT Press

Armstrong, H. (1995): Convergence among Regions in the European Union, 1950-1990. Papers in Regional Science 74; S. 143-152.

Arrow, K. (1972): Gifts and Exchanges. Philosophy and Public Affairs $1 ;$ S. 343-362
Banfield, E. (1958): The Moral Basis of a Backward Society. New York's Free Press

Barro, R. (1991): Economic Growth in a Cross Section of Countries. Quarterly Journal of Economics 106; S. 407-444

Barro, R. (1997): Determinants of Economic Growth: A Cross-Country Empirical Study. Cambridge: MIT Press

Barro, R.; Sala-i-Martin, X. (1992) Convergence. Journal of Political Economy 100:223-51.

Barro, R.; Sala-i-Martin, X. (1995): Economic Growth. New York: McGraw-Hill

Bisin, A.; Verdier, T. (2000): A Model of Cultural Transmission, Voting and Political Ideology. European Journal of Political Economy 16; S. $5-29$

Boix, C.; Posner, D. (1998): Social Capital: The Politics Behind. ECPRnews $9(3)$; S. $13-14$

Bolle, M. (1969a): Einige Bemerkungen zur Theorie des optimalen Wachstums bei endlichem Zeithorizont. Konjunkturpolitik 15

Bolle, M. (1969b): Optimale Staatsausgabenpolitik unter Verwendung quadratischer und zeitminimaler Gütekriterien. Konjunkturpolitik 15

Bourdieu, P. (1983): Ökonomisches Kapital, kulturelles Kapital, soziales Kapital. In: Kreckel, Reinhard (ed.): Soziale Ungleichheiten; Göttingen: Soziale Welt; Sonderband 2

Braithwaite, V;; Levy, M. (1998): Trust and Governance. New York: Russel Sage Foundation

Cattaneo, C. (1957): La citt considerata come principio ideale delle istorie italiane. Scritti storici e geografici 2; S. 383-437

Coleman, J.(1988): Social Capital in the Creation of Human Capital. American Journal of Sociology 94; S. 95-119

Coleman, J.(1990): Foundations of Social Theory. Cambridge; London: The Belknap Press of Harvard University Press

Europäische Kommission (1994): Wettbewerbsfähigkeit und Kohäsion: Tendenzen in den Regionen: Fünfter periodischer Bericht über die sozioökonomische Lage und Entwicklung der Regionen in der Gemeinschaft; Luxemburg: Amt für amtliche Veröffentlichungen der Europäischen Gemeinschaft

Eurostat (1997): Regionen: Statistisches Jahrbuch 1996. Luxemburg: Amt für amtliche Veröffentlichungen der Europäischen Gemeinschaft

Faist, T. (1995): Sociological Theories of International Migration: The Missing Meso-Link. Paper presented at the Meeting of the Theory Group Migration and Development (MAD) Project Hamburg 
Glaeser, E.; Laibson, D.; Scheinkman, J.; Soutter, C. (2000): Measuring Trust. Quarterly Journal of Economics CXV; S. 811-47 (August)

Glaeser, E.; Laibson, D.; Scardote, B. (2000): The Economic Approach to Social Capital. NBER Working Paper 7728

Goldberg, E. (1996): Thinking about how Democracy Works. Politics and Society 24(1); S. 7-18

Granato, J.; Inglehart, R.; Leblang, D. (1996a): Cultural Values, Stable Democracies, and Economic Development: A Reply. American Journal of Political Science 40; S. 680-696

Granato, J.; Inglehart, R.; Leblang, D. (1996b): The Effect of Cultural Values on Economic Development: Theory, Hypotheses, and Some Empirical Test. American Journal of Political Science 40; S. 607-631

Grossman, G.; Helpman. E. (1991): Innovation and Growth in the Global Economy. Cambridge: MIT Press

Guiso, L.; Sapienza, P.; Zingales, L. (2000): The Role of Social Capital in Financial Development. NBER Working Paper 7563

Hansson, P.; Henrekson, M. (1997): Catching-up, Social Capability, Government Size and Economic Growth. In: Bergström, V. (Hrsg.) Government and Growth; Oxford: Clarendon Press; S. 61-148

Haug, S. (1997): Soziales Kapital: Ein kritischer Überblick über den aktuellen Forschungsstand. Arbeitspapier AB II/Nr. 15; Mannheimer Zentrum für Europäische Sozialforschung

Helliwell, J.; Putnam, R. (1999): Education and Social Capital. NBER Working Paper 7121

Hilman, A.; Ursprung, H. (2000): Political Culture and Economic Decline. European Journal of Political Economy 16; S. 189-213

Inglehart, R. (1988): The Renaissance of Political Culture. American Political Science Review 82; S. 1203-1230

Inglehart, R. (1990): Culture Shift in Advanced Industrial Societies. Princeton: Princeton University Press

Inglehart, R. (1997): Modernization and Postmodernization: Cultural, Economic and Political Change in 43 Societies. Princeton: Princeton University Press

Jackman, R.; Miller, R. (1996a): A Renaissance of Political Culture. American Journal of Political Science 40; S. 632-659

Jackman, R.; Miller, R. (1996b): The Poverty of Political Culture. American Journal of Political Science 40; S. 697-716

Jackman, R.; Miller, R. (1998): Social Capital and Politics. Annual Review of Political Science 1; S. 47-73

Knack, S.; Zak, P. (2001): Trust and Growth. Economic Journal; i.E.
Laitin, D. (1995): The Civic Culture at 30. American Political Science Review 89(1); S. 168-173

Lal, D. (1998): Unintended Consequences: The Impact of Factor Endowments, Culture, and Politics on Long-Run Economic Performance. London: MIT Press

LaPalombra, J. (1993): Book Review: Making Democracy Work: Civic Traditions in Modern Italy. Political Science Quarterly 108; S. 549550

Levi, M. (1996): Social and Unsocial Capital: A Review Essay of Robert Putnam's Making Democracy Work. Politics and Society 24(1); S. $45-55$

Levi, M. (1998): A State of Trust. ECPR-news: The News Circular of the European Consortium for Political Research 9(3); S. 14-15

Loury, G. (1977): A Dynamic Theory of Racial Income Differences. In: Wallace, P.A. and A. Le Mund (Eds.) : Women, Minorities, and Employment Discrimination; Lexington: Lexington Books

Loury, G. (1981): Intergenerational Transfers and the Distribution of Earnings. Econometrica 49; S. 843-867

Maraffi, M. (1994): Book Review: Making Democracy Work: Civic Traditions in Modern Italy. American Journal of Sociology 99(5); S. 1348-1349

Maraffi, M. (1998): Voluntary Associations, Political Culture and Social Capital in Italy: A Complex Relationship . ECPR-news 9(3); S. 15-16

Mudambi, R.; Navarra, P. (1999): Political Culture and Foreign Direct Investment: The Case of Italy. Unpublished Paper

Myers, D.(1995): Institutional Performance, Political Culture, and Political Change. Studies in Comparative International Development $30(1)$; S. 84-91

Neven, D.; Gouyette, C. (1995): Regional Convergence in the European Union. Journal of Common Market Studies 33(1); S. 47-65

Ostrom, E. (1998): A Behavioral Approach to the Rational Choice Theory of Collective Action: Presidential Address, American Political Science Association, 1997. American Political Science Review 92(1); S.1-22

Putnam, R.(1995): Bowling Alone: America's Declining Social Capital. Journal of Democracy 6(1); S. 65-78

Putnam, R.(1993): Making Democracy Work: Civic Traditions in Modern Italy. Princeton: Princeton University Press

Putnam, R.(2000): Bowling Alone. The Collapse and Revival of American Community. New York: Simon \& Schuster 
Romer, P. (1986): Increasing Returns and Long-Run Growth. Journal of Political Economy 94(5); S.1002-1037

Romer, P. (1994): The Origins of Endogenous Growth. Journal of Economic Perspectives 8(1); S. 3-22

Sabetti, F. (1996): Path Dependence and Civic Culture: Some Lessons from Italy about Interpreting Social Experiments. Politics and Society 24(1); S. 19-44

Schneider, G.; Plümper, T.; Baumann, S. (2000): Bringing Putnam to the European Regions. On the Relevance of Social Capital for Economic Growth. European Urban and Regional Studies 7; S. 307-317

Solow, R. (1956): A Contribution to the Theory of Economic Growth. Quarterly Journal of Economics 70; S. 65-94

Statistisches Bundesamt. (1997): Statistisches Jahrbuch für das Ausland. Stuttgart: Metzler-Poeschl

Swan, T.(1956): Economic Growth and Capital Accumulation. Economic Record 32; S. 334-361

Swank, D. (1996): Culture, Institutions, and Economic Growth: Theory, Evidence, and the Role of Communitarian Polities . American Journal of Political Science 40; S. 660-679

Tarrow, S. (1996): Making Social Science Work across Time and Space: A Critical Reflection on Robert Putnam's Making Democracy Work: Civic Traditions in Modern Italy . American Political Science Review 90(2); S. 389-397

United Nations (1994): Statistical Yearbook: United Nations Publication No.: E/F.96.XVII.1.New York: United Nations

Van Deth, J.(2000): Interesting but irrelevant: Social Capital and the Saliency of Politics in Western Europe. European Journal of Political Research 37; S. 115-147

Weber, M. [1921] (1972): Wirtschaft und Gesellschaft: Grundriß der verstehenden Soziologie. Tübingen: J.C.B. Mohr

Weil, F.(1994): Book Review of Putnam's Making Democracy Work: Civic Traditions in Modern Italy. Contemporary Sociology; a Journal of Reviews 23(1); S. 373-374

Whiteley, P. (1997): Economic Growth and Social Capital. ECPR workshop on Social Capital and Politico-Economic Performance; University of Bern; Switzerland; April 1997

Whiteley, P. (1998): Economic Growth and Social Capital. ECPR-News $9(3)$; S. $16-18$

Whitely, P.(2000): Economic Growth and Social Capital. Political Studies 48 ; S. 443-466
World Bank (1997): World Economic Indicators (CD-Rom). Washington: World Bank

Zentralarchiv für empirische Sozialforschung Köln (1996): ZA Study Number 2689 Eurobarometer 44.0. Köln: ZA. 